\title{
Identification of Patients at Risk for Ventricular Tachycardia by means of Body Surface Potential Maps
}

\author{
R Bailón*, S Olmos*, BM Horáček\#, P Laguna* \\ * Communications Technology Group, Aragón Institute of Eng. Research, Univ. of Zaragoza, Spain \\ \# Department of Physiology and Biophysics, Dalhousie University, Halifax, NS, Canada
}

\begin{abstract}
In this work we attempted the stratification of patients at risk for VT by means of BSPM recorded during sinus rhythm, based on the evidence that specific arrhythmogenic alterations manifest on body surface potentials. Due to the high dimensionality of BSPM data and the limited number of available patients, a feature extraction step was necessary prior to the classifier design. Feature extraction was performed by means of linear expansions of two different time intervals: $Q R S$ and ST-T complexes. Two approaches were studied: the Karhunen-Love transfom (KLT) and spatio-temporal expansions. A multivariate linear discriminant analysis was applied to the extracted features to classify the study population in two groups: VT and non-VT. Our results showed that spatio-temporal features ( $S E=83 \%, S P=86 \%)$ obtained similar classification results than KLT features ( $S E=78 \%, S P=93 \%$ ) with a lower computational cost. For comparison, a method reported in the literature based on QRST integral maps was implemented, obtaining results within the same range ( $S E=88 \%, S P=72 \%$ ).
\end{abstract}

\section{Introduction}

Ventricular tachycardia (VT) is a major factor of mortality in patients with heart disease. Several approaches have been investigated in order to identify the presence of arrhythmogenic regions in the ventricular myocardium, such as electrophysiological studies, ambulatory monitoring, exercise testing, 12-lead ECG and body surface potential mapping (BSPM). There is some evidence that specific electrophysiological alterations during sinus rhythm are associated with vulnerability to VT and manifested in body surface potentials. Therefore, BSPM analysis is particularly challenging since nearly all noninvasively-available electrocardiographic information can be captured. Previous works showed that subjects at risk for VT have unique map characteristics [1], e.g. the spatial distribution of QRST integrals over the torso was used in [2] to stratify patients at risk for VT.
In this study we attempted to identify patients at risk for VT by means of BSPM recorded during sinus rhythm. The large dimensionality of BSPM data in relation to the limited number of patients of the study population made necessary the reduction of the dimensionality of the original BSPM space prior to the classifier design to avoid the "Hughes phenomena" [3]. First, feature extraction was performed by means of linear expansions; then, a linear discriminant analysis was applied to the reduced feature space to stratify patients at risk for VT. Two approaches of linear expansions were studied: the Karhunen-Loève transform (KLT) and spatio-temporal (TS) expansions, proposed in [4] as a low-complexity approximation to KLT. For comparison purposes, the method in [2], based on the QRST integral, was also implemented.

\section{Materials and methods}

\subsection{Study population}

The study population consisted of 705 patients: 259 normals (noMI/noVT), 69 with no evidence of a previous myocardial infarction (MI) but with a history of spontaneous VT (noMI/VT), 258 with no history of VT but a previous MI (MI/noVT) and 119 with a history of VT and previous MI $(M I / V T)$. The diagnosis of MI was based on non-ECG evidence in the acute phase and the presence of diagnostic 12-lead ECG changes. Patients with a history of VT presented with electrocardiographically documented spontaneous sustained VT in the absence of a reversible cause. Normal subjects had no clinical evidence of arrhythmias or heart disease on history, 12-lead ECG, physical and echocardiographic examination. All subjects were informed of the study's procedures, in accordance with the ethical guidelines approved by the institutional ethics committee.

\subsection{Body surface potential mapping}

BSPMs were recorded at the Victoria General Hospital, Halifax, NS, and at the Foothills Hospital, Calgary, AB, 
Canada, using identical protocols and acquisition systems (sampling rate of $500 \mathrm{~Hz}$, amplitude resolution of $2.5 \mu \mathrm{V}$ ). The BSPM lead array had 120 leads: 3 limb and 117 unipolar chest leads (76 on the front and 41 on the back). BSPMs were recorded during sinus rhythm and supine position for 15 consecutive seconds. QRST complexes were identified and processed to yield a single averaged complex for each lead. The onset and end of ECG waves were determined from the averaged complexes [2].

\subsection{Feature extraction}

Feature extraction from BSPMs was performed by means of linear expansions. Each BSPM was represented by a $N \times L$ matrix $\mathbf{X}$ ( $N$ samples, $L$ channels) which can be decomposed as a linear combination of $N \times L$ elementary matrices $\mathbf{B}_{\mathbf{i j}}$,

$$
\mathbf{X}=\sum_{i=1}^{N} \sum_{j=1}^{L} w_{i j} \mathbf{B}_{\mathbf{i j}}
$$

The elementary matrices $\mathbf{B}_{\mathbf{i j}}$ are often selected in order to pack most of the energy of $\mathbf{X}$ in a small subset of $r \ll N L$ weighting coefficients $w_{i j}$. Two approaches of linear expansions were considered in this work: KLT and TS expansions. Their characteristics and application to multichannel signals, like BSPM, were described in [4]. The basis functions of KLT are built from the dominant eigenvectors of the data covariance matrix. In the case of TS expansions, the basis functions are rank-one matrices of the form $\mathbf{B}_{\mathbf{i j}}=\mathbf{t}_{\mathbf{i}} \mathbf{s}_{\mathbf{j}}{ }^{T}$, being $\mathbf{t}_{\mathbf{i}}$ and $\mathbf{s}_{\mathbf{j}}$ the dominant eigenvectors of the average temporal and spatial covariance matrices, respectively.

The features extracted for BSPMs were the $r$-th dominant coefficients for KLT and TS approaches. The parameter $r$ should be chosen as a trade-off between energy representation and basis function generality. In this work, the rule of thumb $r<(\text { training set size })^{1 / 2}$ was followed to avoid using basis functions overtunned to the training set.

\subsection{Classification}

A stepwise multivariate linear discriminant analysis was independently applied to KLT and TS features to classify subjects into the VT group (noMI/VT $+M I / V T)$ and the non-VT group (noMI/noVT $+M I / n o V T)$. The criterion used in the variable inclusion/rejection was the Wilk's lambda minimization. The number of stepwise selected variables followed the rule \#variables $<$ (smallest group size $)^{1 / 2}$. Discriminant analysis assumes that classification variables are Gaussian within each of the groups. The KolmogorovSmirnov test was used to check the normality of the variables (a $p$-statistic value $>0.05$ assessing the normality of a variable). All statistical analysis was performed using SPSS 11.5.

Classification performance was quantified by the indexes: sensitivity $(S E)$, specificity $(S P)$, positive predictive value $(P+)$, negative predictive value $(P-)$ and exactness $(E X)$.

In this work the study population was divided into two sets: a training set of 200 subjects (50 noMI/noVT, 50 $M I / n o V T, 50 \mathrm{noMI} / \mathrm{VT}, 50 \mathrm{MI} / \mathrm{VT}$ ), used to derive the basis functions (feature extraction) and the discriminant functions (classification), and a test set of 76 subjects (19 noMI/noVT, $19 \mathrm{MI} /$ noVT, 19 noMI/VT, $19 \mathrm{MI} / \mathrm{VT}$ ), used to evaluate classification performance. To further support classification results on the finite-size test set, classification performance was also evaluated on the training set by means of cross-validation (leave-one-out method).

\subsection{QRST-integral}

For comparison purposes, the method in [2] was also implemented. Feature extraction was accomplished in two steps: first, QRST-integral was calculated for each lead as the algebraic sum of the sampled potentials within QRST complex multiplied by the sampling interval of $2 \mathrm{~ms}$; then, KLT was applied to QRST-integral maps. Finally, the $r$-th dominant coefficients constituted the set of features entering a stepwise discriminant analysis, as the explained in Section 2.4.

\section{Results}

In order to study depolarization and repolarization properties, two different time intervals were considered: QRS complex (160 ms centered at the QRS fiducial point, defined as the median center of gravity of the QRS complex among all leads) and ST-T complex (450 ms from QRS end). QRS complex maps were represented by matrices QRS-BSPM dimensioned $81 \times 117$. ST-T complex maps were represented, after decimating the signal by a factor of 5 , by matrices STT-BSPM dimensioned $45 \times 117$. The STT-BSPMs were padded with zeros when ST-T complexes were shorter than $450 \mathrm{~ms}$.

BSPM matrices were normalized to have unit energy $(\|\mathbf{X}\|=1)$, in order to equalize the contribution of all subjects of the training set. The normalization was also performed in the test set because the interesting point was the relative contribution of each basis function to the whole BSPM. In this way, the ECG complexes' energy would not affect transformed coefficients.

Feature extraction was independently applied to QRSBSPMs and STT-BSPMs as explained in Section 2.3. A total of 14 KLT features (denoted QRS-KL and STTKL, respectively) and 14 TS features (QRS-TS and STT- 
TS, respectively) were considered. Stepwise discriminant analysis was independently applied to different variable sets to discriminate between the VT and non-VT groups. The variable sets were the QRS-KL, STT-KL, QRS-TS and STT-TS features. Two other variable sets were also considered, QRS-STT-KL and QRS-STT-TS, containing the most significant features from both intervals. A maximum number of 10 variables was allowed in the discrimination (Section 2.4).

All variables had normal distribution according to Kolmogorov-Smirnov test, but for the most dominant STT-TS feature within the non-VT group. An arcsin transformation was performed to correct its lack of normality but classification results did not change.

The EX achieved in the test set by the different variable sets is shown in Fig. 1 as a function of the number of variables used. It can be appreciated that there is a threshold above which EX is not increased, but even decreased, as using a higher number of variables.

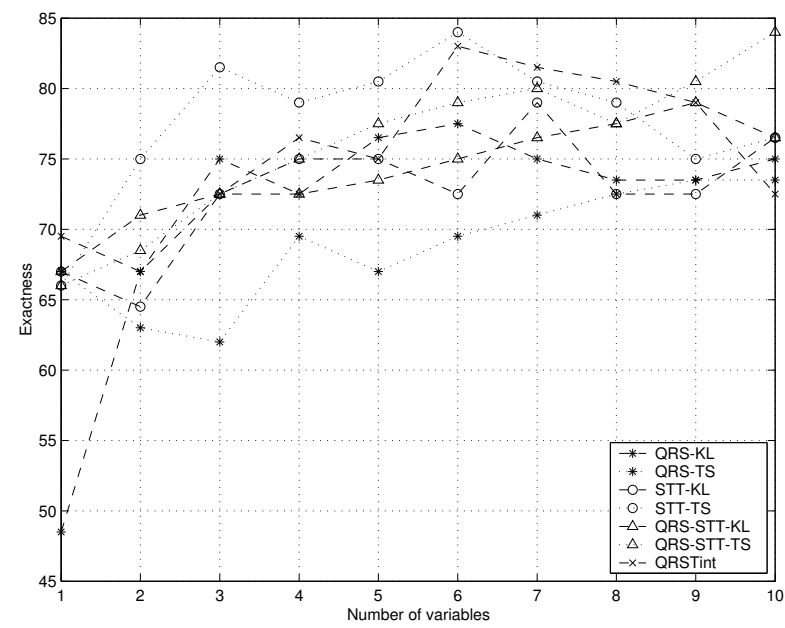

Figure 1. Exactness as a function of the number of variables in the test set.

Classification indexes achieved in the test by each variable set, when using the variables giving the maximum EX, are shown in Table 1.

The QRST-integral used in [2] was applied to our study population as explained in Section 2.5. Exactness achieved in the test set as a function of the number of variables can be seen in Fig.1, where the existence of a threshold is also observed in this case.

Cross-validation classification results achieved by the different variable sets in the training set are shown in Table 2, mostly supporting the results obtained in the test set.
Table 1. Classification results in the test set

\begin{tabular}{|c|c|c|c|c|c|}
\hline Variable set & SE & SP & P+ & P- & EX \\
\hline QRS-KL(6) & 68 & 87 & 84 & 73 & 77 \\
\hline QRS-TS(9) & 68 & 79 & 76 & 71 & 73 \\
\hline STT-KL(7) & 89 & 68 & 74 & 87 & 79 \\
\hline STT-TS(6) & 92 & 76 & 80 & 91 & 84 \\
\hline QRS-STT-KL(9) & 68 & 89 & 87 & 74 & 79 \\
\hline QRS-STT-TS(10) & 76 & 92 & 91 & 80 & 84 \\
\hline QRSTintegral(6) & 66 & 100 & 100 & 75 & 83 \\
\hline
\end{tabular}

The number in brackets represents the number of variables used.

Table 2. Cross-validation classification results in the training set

\begin{tabular}{|c|c|c|c|c|c|}
\hline Variable set & SE & SP & P+ & P- & EX \\
\hline QRS-KL & 79 & 93 & 92 & 82 & 86 \\
\hline QRS-TS & 81 & 83 & 83 & 81 & 82 \\
\hline STT-KL & 76 & 80 & 79 & 77 & 78 \\
\hline STT-TS & 76 & 84 & 83 & 78 & 80 \\
\hline QRS-STT-KL & 78 & 93 & 92 & 81 & 85 \\
\hline QRS-STT-TS & 83 & 86 & 86 & 83 & 84 \\
\hline QRST-integral & 88 & 72 & 76 & 86 & 80 \\
\hline
\end{tabular}

\section{Discussion and conclusions}

BSPM constitutes an encouraging technique in noninvasive cardiology since nearly all available electrocardiographic information can be captured. In this work we dealt with the identification of patients at risk for VT by means of features extracted from BSPM recorded during sinus rhythm. In an attempt of designing the optimal classifier using all available information, we had previously implemented a parametric-linear classifier, a parametric-quadratic classifier and a nonparametric classifier, based on [3]. Our results showed that the number of subjects in the training set used to design the classifier was insufficient given the dimensionality of the data because of the so-called "Hughes phenomena" [3]. As a consequence the classifiers were overfitted to the particular study population and failed in prospective populations. Therefore, a feature extraction step was necessary to reduce BSPM dimensionality while maintaining the important diagnostic information. Then, the classifier was designed from the reduced feature space.

Feature extraction was performed by means of linear expansions. The Fourier transform had been studied to reduce BSPM dimensionality [5], but the requirement of equally spaced samples constituted a restrictive limitation in the spatial domain. Two approaches were considered in this study: KLT and TS. KLT had been previously applied to BSPMs in other works to eliminate spatial [2] and temporal redundancies [6]. One of the limitations of 
the KLT is the high computational load and complexity required for computing the basis functions and the transformed coefficients. In [4] TS was proposed as a low-complexity approximation of the KLT, getting similar energy packing performances in a 12-lead ECG database. KLT and TS were compared in this work in terms of diagnostic classification performance. The number of subjects in the training set limited to 14 the order of KLT and TS basis function estimated avoiding the lack of generality. Higher order basis functions would collect particular details from the training set instead of the general behaviour of BSPMs.

The choice of a linear classifier was based on its simplicity, relative insensitivity to a limited-size training set and good performance even when unfulfilled underlying assumptions. Results show the existence of a threshold in the number of variables used by the classifier above which classification performance did not longer improved. Classification performance achieved by KLT and TS features were within the same range of exactness, slightly varying $(<5 \%)$ depending on the population and the variables used in the classifier. This extends the findings in [4], suggesting that TS can be used as an approximation of KLT not only for energy packing but also for diagnostic classification in BSPM analysis. In this case, the main advantage of TS over KLT is the complexity reduction in basis functions' computation (from $O\left((N L)^{3}\right)$ to $O\left(N^{3}+\right.$ $\left.L^{3}\right)$ ). The complexity in estimating the transformed coefficients is lower for TS than for KLT if $r>(N+L)$, which is not the case in this work.

Classification results achieved by depolarization and repolarization features corroborate the previous finding that vulnerability to VT alters depolarization [7] and repolarization properties [8], and suggest that these alterations may be correlated since only a slight improvement in classification performance is achieved when depolarization and repolarization periods are jointly considered.

QRST-integral maps have been widely analyzed as an indicator of vulnerability to VT [1, 2]. In [2] the spatial distribution of QRST-integral maps were reported to identify patients at risk for VT with a SE of $90 \%$ and a SP of $80 \%$. In our study population classification performance achieved by QRST-integral maps was slightly lower ( $\mathrm{SE}=88 \%, \mathrm{SP}=72 \%$ ), within the same range of KLT and $\mathrm{TS}$ features $(\mathrm{SE} \approx 80, \mathrm{SP}=\approx 90$ in average). These results suggest that the relevant information for identifying patients at risk for VT is contained in the spatial distribution of BSPM rather than in the temporal waveform, since the spatial distribution of a single measurement per beat (QRST integral) obtained similar results than when the whole QRST complex was considered.

The main limitation of this work is the insufficient number of patients available, given the data dimensionality, to design the classifier [3]. Therefore, it was extremely important to derive a reduced feature space without lost of diagnostic information and avoiding the overtunning.

In a futher study, the discriminant KLT and TS features selected by the stepwise procedure should be physiologically interpreted to understand how the arrhythmogenic substrate may manifest through them.

Acknowledgements This work was supported by projects TIC 2001-2167-CO2-02 from MCYT and P075/2001 from CONSID-DGA.

\section{References}

[1] Gardner M, Montague T, Armstrong C, Horáček B, Smith E. Vulnerability to ventricular arrhythmia: Assessment by mapping of body surface potential. Circulation 1986;73:684692.

[2] Hubley-Kozey C, Mitchell L, Gardner M, Warren J, Penney C, Smith E, Horáček B. Spatial features in body-surface potential maps can identify patients with a history of sustained ventricular tachycardia. Circulation 1995;92:1825-1838.

[3] Fukunaga K. Introduction to statistical pattern recognition. San Diego, California: Academic Press, 1990.

[4] Olmos S, Martínez J, Sörnmo L. Spatio-temporal linear expansions for repolarization analysis. In Computers in Cardiology. Memphis, TN, USA: IEEE Computer Society Press, 2002; 689-692.

[5] Monro D, Guardo R, Bourdillon P, Tinker J. A Fourier technique for simultaneous electrocardiographic surface mapping. Cardiovasc Res 1974;8:688-700.

[6] Evans A, Lux R, Burgess M, Wyatt R, Abildskov J. Redundancy reduction for improved display and analysis of body surface potential maps. II Temporal compression. Circ Res 1981;49:197-203.

[7] Faugère G, Savard P, Nadeau R, Derome D, Shenasa M, Page $\mathrm{P}$, Guardo R. Characterization of the spatial distribution of late ventricular potentials by body surface mapping in patients with ventricular tachycardia. Circulation 1986;74:1323-1333.

[8] Okin P, Devereux R, Fabsitz R, Lee E, J.M.Galloway, Howard B. Principal component analysis of the $T$ wave and prediction of cardiovascular mortality in american indians. The Strong Heart Study. Circulation 2002;105:714-719.

Address for correspondence:

R. Bailón.

Dep. Ing. Electrónica y Comunicaciones

María de Luna, 1. 50015-Zaragoza (SPAIN)

E-mail: rbailon@unizar.es 